\title{
Correlative Analysis of GRBs detected by Swift, Konus and HETE
}

\author{
H. A. Krimm*, ${ }^{*}$, S. D. Barthelmy*, N. Gehrels*, D. Hullinger** T.

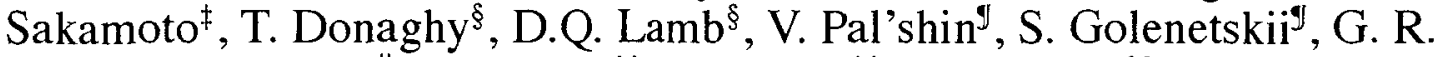 \\ Rickerll, J-L Atteia ${ }^{\dagger \dagger}$, N. Kawai ${ }^{\ddagger \ddagger}$ and G. Sato ${ }^{\S \S, \text { II }}$ \\ *NASA Goddard Space Flight Center, Greenbelt, Maryland, 20771, USA \\ $\dagger$ Universities Space Research Association, 10211 Wincopin Circle, Ste 500, Columbia, MD 21044 \\ ${ }^{* *}$ University of Maryland, College Park, MD, USA \\ ${ }^{\ddagger}$ National Research Council, Washington, DC, USA \\ $\S$ University of Chicago, Chicago, IL USA \\ Ioffe Physico-Technical Institute, Laboratory for Experimental Astrophysics, 26 \\ Polytekhnicheskaya, St Petersburg 194021, Russian Federation \\ "Massachusetts Institute of Technology, Cambridge, MA USA, \\ ${ }^{\dagger \dagger}$ Observatoire Midi-Pyrenees, Toulouse, France \\ Tokyo Institute of Technology, Tokyo, Japan \\ $\$$ Institute of Space and Astronautical Science, JAXA, Kanagawa, Japan \\ II University of Tokyo, Tokyo, Japan
}

\begin{abstract}
Swift has now detected a large enough sample of gamma-ray bursts (GRBs) to allow correlation studies of burst parameters. Such studies of earlier data sets have yielded important results leading to further understanding of burst parameters and classifications. This work focusses on seventeen Swift bursts that have also been detected either by Konus-Wind or HETE-II, providing high energy spectra and fits to $E_{\text {peak }}$. Eight of these bursts have spectroscopic redshifts and for others we can estimate redshifts using the variability/luminosity relationship. We can also compare $E_{\text {peak }}$ with $E_{\text {iso }}$, and for those bursts for which a jet break was observed in the afterglow we can derive $E g$ and test the relationship between $E_{\text {peak }}$ and $E_{\gamma}$. For all bursts we can derive durations and hardness ratios from the prompt emission.
\end{abstract}

Keywords: gamma-ray bursts, gamma-ray telescopes

PACS: $98.70 . \mathrm{Rz}, 95.55 . \mathrm{Ka}$

\section{METHODOLOGY}

For the bursts used in this study (Table 1 ) we derived $E_{\text {peak }}, E_{i s o}$ and $L_{i s o}$ by the following method. First, for each burst we carried out a joint fit to the BAT and either the Konus or HETE time-averaged spectral data, depending which other instrument detected the burst. Fits were made to both the GRBM (Band) model and a power law model with an exponential cut-off. If the high energy photon index $\beta$ was constrained in the Band model fit, then we used the Band model parameters $\left(\alpha, \beta, E_{\text {peak }}\right)$ in our further analysis; if there was no constraint on $\beta$, then we used the cut-off power law parameters $\left(\alpha, E_{\text {peak }}\right)$ and set $\beta=-10$. In either case, $E_{\text {peak }}$ was converted to the source frame.

Next we derived a spectrum for the peak $1 \mathrm{~s}$ ( $L_{\text {iso }}$ calculation) and total burst ( $E_{\text {iso }}$ calculation ) which we fit to the GRBM model. To allow direct comparison with the results of [1] for $L_{i s o}$, the 1-s peak flux was defined in the energy range $30-10,000 \mathrm{keV}$. 
TABLE 1. GRBs detected by Swift/BAT as well as either Konus-Wind (K) or HETE (H)

\begin{tabular}{|c|c|c|c|c|c|c|}
\hline GRB & $\mathrm{z}$ & $\alpha$ & $\beta$ & $E_{\text {peak }}(\mathbf{k e V})$ & $E_{i s o}\left(10^{52} \mathrm{erg}\right)$ & $L_{i s o}\left(10^{52} \mathrm{erg} / \mathrm{s}\right)$ \\
\hline $041223 \mathrm{~K}$ & - & $-0.83 \pm 0.05$ & - & $337.6_{-24.2}^{+27.7}$ & - & - \\
\hline $050215 \mathrm{~B} \mathrm{H}$ & _- & $-1.29 \pm 0.75$ & - & $28.6 \pm 9.4$ & - & - \\
\hline 050219B K & - & $-1.05 \pm 0.07$ & - & $162.8 \pm 12$ & - & - \\
\hline $050326 \mathrm{~K}$ & - & $-0.78 \pm 0.16$ & $-2.48_{-0.63}^{+0.24}$ & $206.4_{-27 .}^{+32.8}$ & - & - \\
\hline $050401 \mathrm{~K}$ & 2.9 & $-0.90 \pm 0.30$ & $-2.55_{-0.44}^{+0.62}$ & $117.5 \pm 18$ & $41.2 \pm 4.0$ & $14.9 \pm 1.1$ \\
\hline 050416B K & - & $-1.19_{-0.40}^{+0.66}$ & - & $115.5_{-43.4}^{+157}$ & - & - \\
\hline $050418 \mathrm{~K}$ & - & $\begin{array}{l}-1.19_{-0.37}^{+0.69} \\
-10\end{array}$ & - & $55.2 \pm 12.5$ & - & - \\
\hline $050525 \mathrm{~A} \mathrm{~K}$ & 0.606 & $-1.01 \pm 0.11$ & $-3.26_{-0.41}^{+0.23}$ & $81.2 \pm 2.3$ & $2.3 \pm 0.09$ & $7.75 \pm 0.75$ \\
\hline $050603 \mathrm{~K}$ & 2.821 & $-1.03 \pm 0.11$ & $-2.03_{-0.29}^{+0.17}$ & $343.7 \pm 87$ & $61.8 \pm 3.1$ & $67.8 \pm 3.3$ \\
\hline $050713 \mathrm{~K}$ & - & $-1.32 \pm 0.06$ & - & $445_{-87}^{+129}$ & - & - \\
\hline $050717 \mathrm{~K}$ & - & $-1.04 \pm 0.05$ & - & $2400_{-570}^{+780}$ & - & - \\
\hline 050820A K & 2.612 & $-1.25 \pm 0.15$ & - & $246.0_{-66}^{+127}$ & 80 & $2.70 \pm 0.16$ \\
\hline $050824 \mathrm{H}$ & 0.83 & - & - & $<12.7$ & $0.179_{-0.083}^{+6.8}$ & - \\
\hline $050904 \mathrm{~K}$ & 6.29 & $-1.11 \pm 0.10$ & - & $436_{-151}^{+335}$ & $140^{-0.083}$ & $10.6 \pm 1.1$ \\
\hline $050922 \mathrm{C} \mathrm{H}$ & 2.198 & $-0.95 \pm 0.11$ & - & $196.8_{-37}^{+64}$ & $4.64 \pm 0.12$ & $5.3 \pm 0.37$ \\
\hline $051008 \mathrm{~K}$ & - & $-1.07 \pm 0.03$ & - & $888 \pm 669$ & - & - \\
\hline 051109A K & 2.346 & $-1.38 \pm 0.33$ & - & $139.5_{-45}^{+116}$ & 2.4 & $2.80 \pm 0.40$ \\
\hline
\end{tabular}

For comparison with the results of [2] and [3], the total fluence was over 1-10,000 keV. We then used a simple cosmological model $\left(H_{0}=70 \mathrm{~km} / \mathrm{s}, \Omega_{m}=0.3\right.$ and $\left.\Omega_{L}=0.7\right)$ to derive $E_{i s o}$ and $L_{i s o}$ from the calculated flux.

\section{COMPARISON TO PUBLISHED RELATIONS}

\section{Yonetoku Relation}

For seven of the Swift/Konus/HETE bursts in the study set, we have a measurement of both $E_{\text {peak }}$ and a spectroscopic redshift. For these bursts we can compare the parameters derived in this work to the results presented by Yonetoku et al. [1], who compare $E_{\text {peak }}$ in the source frame to the peak luminosity of the burst. These data are shown in the left-hand plot of Figure 1.

We can see that while our results do not cluster as tightly as the data points Yonetoku et al. [1] use, all of the points save for GRB 050904 (see below) lie in or very near the error range of these results. Since three of the points are above the line and four below, there does not appear to be a systematic error in our calculations of either $E_{\text {peak }}$ or $L_{i s o}$. Note that GRB 050820A is plotted twice. Since the BAT data was cut off before the largest peak, when $L_{i s o}$ is derived from the BAT data alone (lower point), $L_{i s o}$ is underestimated. We used the Konus light curve which includes both peaks to derive a scale factor; this provides the upper point, which is closer to the relation.

The most extreme outlier is GRB 050904, the highest redshift burst seen by Swift (z=6.29). Though it had a high rest frame $E_{\text {peak }}$, it was also fairly low luminosity. Thus we urge caution in interpreting the Yonetoku et al. [1] relation for high redshift GRBs. 

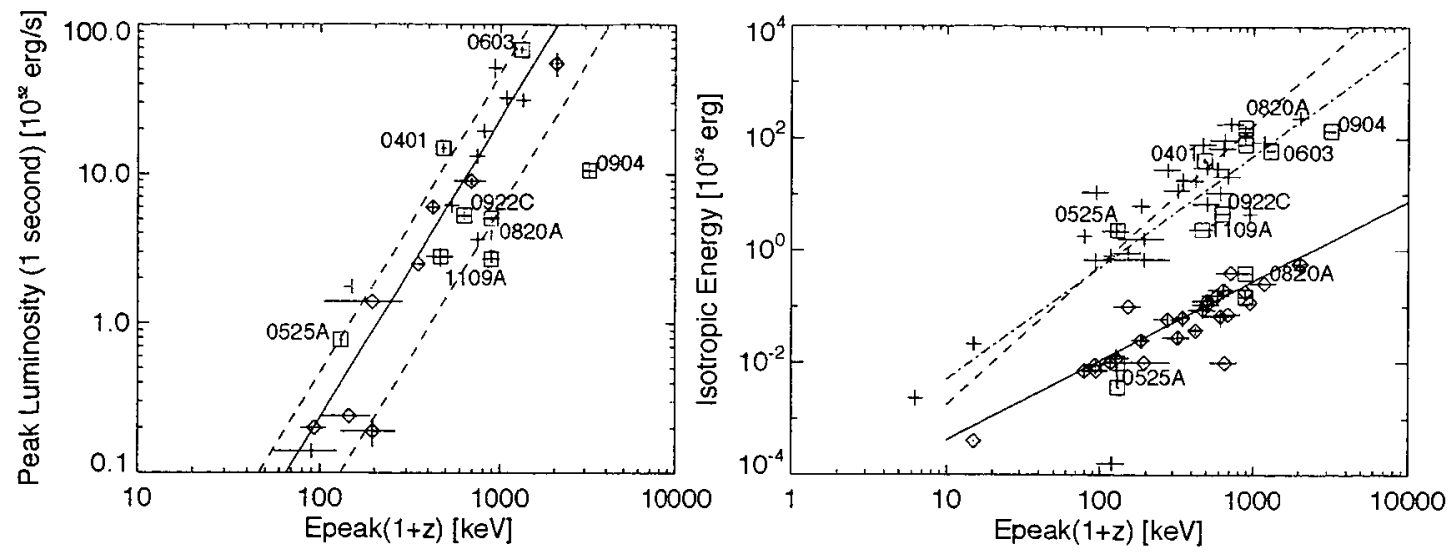

FIGURE 1. Comparison to the Yonetoku, Ghirlanda and Amati relations. Left: Yonetoku. Crosses: BATSE bursts; diamonds: BeppoSAX bursts; squares: Swift bursts. Swift bursts are identified by the month and day (all 2005) of the burst. The solid line shows the relationship derived by Yonetoku et al. [1]: $\log \left(L_{\text {iso }}\right)=2.0 \log \left(E_{\text {peak }}(s r c)\right)-4.63$ and the dashed lines use the \pm 1 s uncertainties in the intercept, keeping the slope constant. Right: Ghirlanda/Amati. Comparison to the Ghirlanda and Amati relations. Data from [3]: crosses are $E_{\text {iso }}$; diamonds are $E_{\gamma}$ The BAT/Konus/HETE points are shown as squares. The solid line shows the relationship derived by [3]: $\log \left(E_{\gamma}\right)=1.42 \log \left(E_{\text {peak }}\right)-4.80$. The dashed line is the fit that [3] derived between $E_{\text {iso }}$ and $E_{\text {peak }}$ and the dot-dash line is the relationship derived by [2].

\section{Amati and Ghirlanda Relations}

For the same set of bursts discussed above we can compare the parameters derived in this work to the results presented in earlier analyses. Amati et al. [2] compare $E_{\text {peak }}$ in the source frame to the total isotropic luminosity of the burst, assuming isotropic emission $\left(E_{i s o}\right)$. Ghirlanda, Ghisellini \& Lazzati [3], confirm this relation and derive a separate, but related relationship which assumes beamed emission, $E_{\gamma}=(1-\cos \theta) E_{i s o}$, where $\theta$ is the jet collimation angle. The data derived from [3] are shown in the right-hand panel of Fig 1 .

We are able to confirm the $E_{\text {peak }}-E_{\text {iso }}$ ([2]) relation with our data. Again there is no evidence for a systematic error in our calculations. There are however, several outliers. As discussed above, correcting for the missing flux from GRB 050820A brings this data point very close to the parameterization in [3]. We note that GRB 050904 is also below the relations. Since we are integrating the entire burst, time dilation should not have an effect and we see that our point is closer to the relation than it is in the left-hand panel (Yonetoku). However, we still may not be properly accounting for all of the flux from this burst. There were several spectrally hard X-ray flares at later times that may be part of the prompt emission [5]. Thus $E_{i s o}$ for this burst should be treated as a lower limit. We have no explanation for the other two outliers, GRB 050922C and GRB 051 109A.

Ghirlanda, Ghisellini \& Lazzati [3] et al found a tighter relationship between $E_{\text {peak }}$ and $E_{\gamma}\left(E_{i s o}\right.$ corrected for the jet opening angle). Of the seven Swift/Konus/HETE bursts studied, only two, GRB 050525A and GRB 050820A, have a verified jet break. For GRB 050525A, Blustin et al. [6] reports two possible jet break times and corresponding opening angles: $t_{j} \sim 0.15 \mathrm{~d}\left(3.2^{\circ}\right)$ and $t_{j} \sim 0.6 \mathrm{~d}\left(5.4^{\circ}\right)$. These are both indicated in the 


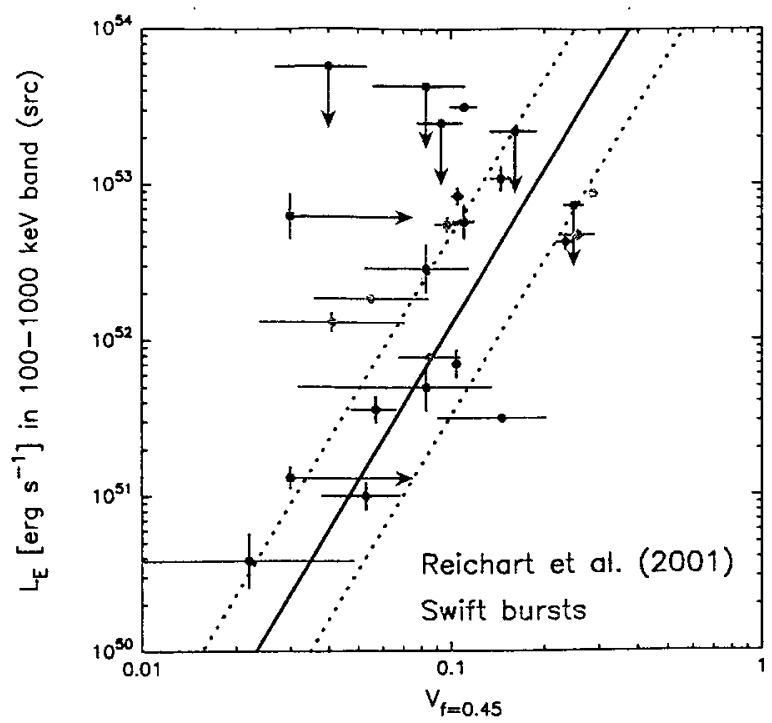

FIGURE 2. Comparison to the relation in [4]. Blue: Reichart's original points; red: Swift'BAT points.

figure, the higher point represents the larger opening angle and is more consistent with the points from [3]. The data for GRB 050820A is from Osborne et al. [7] and the two points are due to the scaling discussed above.

\section{Reichart V-L Relation}

Reichart et al. [4] derived a possible luminosity estimator for GRBs based on the variability of their light curves. We have repeated their analysis here for those GRBs in the study sample which had high enough SNR for the variability to be estimated. Of the seven sample bursts with known redshifts, we were able to derive variability for all save for GRB 050904. The six remaining points are plotted in Figure 2. The variability shown is the weighted average of BAT channels 2, 3, and 4 (whenever the SNR in channel 4 is large enough). These correspond to energy bands (25-50, 50-100 and 100-350 keV). This is close, although not perfect match to the energy ranges used by Reichart et al. [4]. We see in the figure that there is a reasonable match between the Swift data points and the original points. As in the $E_{\text {peak }}$ relations discussed above, there is no sign of a systematic error in our calculations.

\section{REFERENCES}

1. Yonetoku, D., et al, $A p J, 609,935$, (2004).

2. Amati, L., et al., $A \& A, \mathbf{3 9 0}, 81,(2002)$.

3. Ghirlanda, G, Ghisellini, G., \& Lazzati, D., ApJ, 616, 331, (2004).

4. Reichart, D.E., et al, $A p J$, 552, 57, (2001).

5. Cummings, J. et al, these proceedings (2006).

6. Blustin, A. J., in press astro-ph/0507515(2006).

7. Osborne, J. et al, these proceedings (2006). 